\title{
Cartografia da vulnerabilidade socioambiental: convergências e divergências a partir de algumas experiências em Portugal e no Brasil
}

\author{
Cartography of the socio-environmental vulnerability: convergences \\ and differences from some experiences in Portugal and Brazil
}

Maria Isabel Castreghini Freitas ${ }^{[a]}$, Lúcio Cunha ${ }^{[b]}$

[a] Engenheira cartógrafa, professora do Departamento de Planejamento Territorial e Geoprocessamento (IGCE) da Universidade Estadual Paulista (Unesp), Rio Claro, SP - Brasil, e-mail: ifreitas@rc.unesp.br

[b] Geógrafo, professor catedrático do Departamento de Geografia e Centro de Estudos de Geografia e Ordenamento do Território (CEGOT) da Universidade de Coimbra (UC), Coimbra - Portugal, e-mail: luciogeo@ci.uc.pt

\section{Resumo}

Com este estudo, buscamos contextualizar o tema vulnerabilidade socioambiental no cenário mundial, destacando similaridades e discrepâncias nas suas concepções em Portugal e no Brasil. Na literatura que trata de vulnerabilidade já está consagrada a contribuição das Geotecnologias e do seu potencial para a modelagem de aspectos físicos e socioeconômicos, para a prevenção, mitigação e enfrentamento de manifestações de riscos, sejam eles naturais, tecnológicos ou mistos. Este artigo tem como objetivo discutir os referenciais metodológicos dos estudos de vulnerabilidade, bem como os resultados de aplicação na modelagem de dados socioeconômicos e ambientais no contexto da região Centro de Portugal e do estado de São Paulo, Brasil. A metodologia para a análise da vulnerabilidade dessas regiões baseou-se na avaliação quantitativa da capacidade de resistência e de resiliência de populações e de territórios. Os resultados apresentam-se coerentes com as realidades socioambientais das áreas de estudo e refletem a complexidade quando do enfrentamento e recuperação de situações de riscos para o caso de populações e territórios menos providos de condições econômicas e de infraestruturas urbanas, seja no Brasil ou em Portugal.

Palavras-chave: Vulnerabilidade socioambiental. Cartografia. Metodologia quantitativa. Portugal. Brasil.

\section{Abstract}

In this study we intent to contextualize the topic environmental vulnerability on the world stage, highlighting similarities and differences in their conceptions in Portugal and Brazil. In the literature about the vulnerability is already established the contribution of Geotechnologies, especially the Systems of Remote Sensing and Orbital Imaging as well as Geographic Information Systems (GIS) and its potential for modelling physical and socioeconomic aspects for the prevention, mitigation and facing risk manifestations, whether natural, technological or mixed. This paper aims to discuss the methodological framework of vulnerability studies and the 
results of application in the modelling of socioeconomic and environmental data in the context of the Region Centre of Portugal and the State of Sao Paulo, Brazil. The methodology for analysing the vulnerability of these regions was based on quantitative studies of the capacity of resistance and resilience of populations and territories. The results were consistent with the socio-environmental realities of the study areas and reflect the complexity of facing and recovery of risk situations in case of territories and populations under low economic conditions and urban infrastructure, whether in Brazil and Portugal.

Keywords: Socio-environmental vulnerability. Cartography. Quantitative methodology. Portugal. Brazil.

\section{Vulnerabilidade socioambiental: conceitos fundamentais e principais diretrizes metodológicas}

O aumento progressivo da população urbana, o consequente aumento dos diferentes tipos de riscos urbanos (naturais, tecnológicos ou mistos) e a deterioração dos ecossistemas tornam imprescindível o traçado de ações integradas, envolvendo governo e parceiros locais, para definir políticas que transformem o conhecimento já consolidado em ações concretas, em larga escala, que resultem em benefícios para toda a sociedade.

Nesse sentido, a United Nations - International Strategy For Disaster Reduction (UN-ISDR, 2009) destaca algumas necessidades urgentes que devem nortear as políticas dos países no que concerne a riscos naturais e tecnológicos:

- a harmonização urgente da redução de riscos de desastres e a adaptação às mudanças climáticas no contexto da redução da pobreza e do desenvolvimento sustentável;

- a redução dos riscos para comunidades locais, por meio de parcerias colaborativas baseadas no reconhecimento da dependência mútua do governo central e local e dos atores da sociedade civil;

- a transformação de ações isoladas e de projetos-piloto em implementações plenas em segurança, saúde e educação e planos de ação concretos para escolas e hospitais seguros, para atendimento a eventos relacionados com desastres;
- o aumento da escala das ações e financiamentos para o investimento na redução dos riscos;

- a constituição de um sistema de estratégias de suporte ao governo e a organizações da sociedade civil, com capacidade regional, para coordenação e suporte a eventos de risco.

Tais orientações têm como referência o Quadro de Ações de Hyogo (HFA) ${ }^{1}$, que definiu 5 ações prioritárias com vistas à prevenção de riscos, conforme a UN-ISDR (2005). Dentre estas, merece destaque a ação prioritária 4, que indica a urgência de reduzir os riscos relacionados às mudanças das condições socioeconômicas e ambientais, que são as bases para o mapeamento da vulnerabilidade. Como as questões relacionadas com os riscos estão na pauta de discussões dos principais organismos internacionais, fica difícil para os governos e os diferentes estratos de nossa sociedade ficarem alheios aos problemas relacionados com as manifestações de eventos perigosos.

0 conceito de vulnerabilidade é absolutamente indissociável do conceito de risco (REBELO, 2010). Dito de outra forma, para que se possa falar de risco é necessário que haja também vulnerabilidade, ou seja, que os processos eventualmente perigosos (sismos, inundações, incêndios, por exemplo) afetem, direta ou indiretamente, individual ou coletivamente, o ser humano, na sua saúde, nos seus bens ou nos modos de funcionamento das instituições em que se enquadram, na economia, na sociedade e na cultura. Para alguns autores, o conceito de vulnerabilidade deve estender-se também aos elementos

\footnotetext{
1 Hyogo Framework for Action (2005-2015) é um plano de 10 anos para tornar o mundo mais seguro contra riscos naturais, que foi aprovado por 168 Estados-Membros das Nações Unidas, em 2005, na Conferência Mundial de Redução de Desastres que ocorreu em Kobe, Hyogo - Japão.
} 
mesológicos ou ambientais (água, ar, florestas, por exemplo), ou seja, a um conjunto de elementos que, apesar de exteriores ao ser humano, em muito contribuem para a sua qualidade de vida.

0 conceito de vulnerabilidade socioambiental, mais utilizado no Brasil do que em Portugal, aplica-se exatamente a esta vulnerabilidade de sentido muito amplo e que, na acepção de S. Cutter (2011, p. 60), corresponde ao "potencial para a perda". Segundo a autora, a vulnerabilidade inclui tanto os "elementos de exposição ao risco" como os "fatores de propensão às circunstâncias que aumentam ou reduzem as capacidades da população, das infraestruturas ou dos sistemas físicos para responder e se recuperar de ameaças ambientais".

Cutter, Boruff e Shirley (2003), tomando por base as reflexões de diversos autores, consideram que as pesquisas em vulnerabilidade podem ter três abordagens fundamentais: a da identificação das condições que tornam pessoas e lugares vulneráveis aos eventos naturais perigosos; aquelas que consideram a vulnerabilidade como condição social, ou seja, uma medida da resistência ou resiliên$\mathrm{cia}^{2}$ às catástrofes naturais, e aquelas que integram o potencial de exposição e a resiliência social num dado local ou região.

There are three main tenets in vulnerability research: the identification conditions that make people or places vulnerable to extreme natural events, an exposure model (Burton, Kates, and White, 1993; Anderson, 2000); the assumption that vulnerability is a social condition, a measure of societal resistance or resilience to hazards (Blaikie et al., 1994; Hewitt, 1997); and the integration of potential exposures and societal resilience with a specific focus on particular places or regions (Kasperson, Kasperson, and Turner, 1995; Cutter, Mitchell, and Scott, 2000) (CUTTER; BORUFF; SHIRLEY, 2003, p. 243-244).

Na busca por uma definição de vulnerabilidade merecem destaque Blaikie et al. (1994), os quais consideram vulnerabilidade como o conjunto das características de uma pessoa ou grupo, em termos de sua capacidade de antecipar, enfrentar, resistir e se recuperar do impacto de um fenômeno perigoso. Os autores consideram que este conceito envolve uma combinação de fatores que determinam o modo e o grau em que a vida e o sustento dos indivíduos são colocados em perigo por um evento identificável da natureza ou da sociedade.

Na perspectiva de Cutter (1996), a vulnerabilidade corresponde a um conceito complexo, sob o qual advêm dimensões sociais, econômicas, políticas e culturais, cuja definição tem sido abordada também em perspectivas epistemológicas muito diversas na ecologia política, ecologia humana, ciências físicas e análise espacial. Em recente trabalho, Cutter (2011) se refere à necessidade de uma abordagem integradora e interdisciplinar para o estudo da vulnerabilidade social e/ou socioambiental e essa necessidade decorre, sobretudo, da complexidade das interações entre os sistemas naturais, sociais, econômicos e culturais em jogo. A autora elege, também, como princípio fundamental do que chama a "ciência da vulnerabilidade", o "requisito do conhecimento geoespacial da investigação, com base nos locais [...] Todos os desastres são locais e as respostas também o são" (CUTTER, 2011, p. 61). É também a importância da escala local que coloca os aglomerados populacionais e, em particular, as cidades, apesar das suas diferenciações e mesmo das suas contradições internas, como objetos particulares do estudo das vulnerabilidades.

Apesar deste entendimento, o estudo da vulnerabilidade, considerada como um produto da exposição aos processos perigosos e dos fatores de predisposição (a vulnerabilidade social, em sentido restrito), pode e tem sido realizado em diferentes escalas, que vão do nacional ao local, passando pelo regional. Em todas as escalas, a cartografia dos resultados é fundamental, até para que se possa juntar à cartografia dos processos perigosos (suscetibilidade e perigosidade ${ }^{3}$ ) para se atingir uma verdadeira cartografia de risco.

\footnotetext{
2 Conceito originário da Física que diz respeito à propriedade de alguns materiais em acumular energia quando submetidos a estresse sem que ocorra sua ruptura. No contexto desses estudos, conforme Marandola Jr. e Hogan (2005, p. 32-33), se reporta "a capacidade individual e dos sistemas sociais para absorver os efeitos das flutuações ambientais extremas".

3 Perigosidade é um termo adotado em Portugal para indicar a possibilidade (ou probabilidade) de acontecer um evento perigoso. Similar à periculosidade.
} 
E, quer na cartografia da perigosidade, quer na da vulnerabilidade, as geotecnologias e, particularmente, os Sistemas de Informação Geográfica (SIG) são fundamentais, não apenas como importantes instrumentos de produção cartográfica, mas, sobretudo, por sua capacidade de cruzamento da informação e de modelagem dos diferentes componentes do risco.

No âmbito da Geografia, muitos são os trabalhos que visam à modelagem de fenômenos relativos à sociedade e ao ambiente por meio das geotecnologias. De acordo com Marandola Jr. e Hogan (2004), os primeiros trabalhos geográficos com essa abordagem surgiram nos anos de 1980, pautados nos fenômenos naturais que causavam danos e expunham as populações ao perigo.

Os "natural hazards", ou perigos naturais, têm exigido grande esforço e apreensão por parte de pesquisadores envolvidos com ações de planejamento e gestão e com a relação do homem com seu ambiente. [...] 0 prognóstico da probabilidade de aqueles fenômenos [riscos naturais] ${ }^{4}$ ocorrerem era fundamental naquele contexto. Nesse sentido, os geógrafos desenvolveram largamente o que chamavam de risk assessment (avaliação do risco): avaliação do risco de ocorrer um perigo [um acidente ou catástrofe] ${ }^{5}$ em determinado local (MARANDOLA Jr; HOGAN, 2004, p. 31).

Nesse contexto, começa a ganhar maior expressão esse tipo de pesquisa envolvendo riscos naturais, fornecendo subsídios para a introdução, no meio científico, do conceito de vulnerabilidade. Segundo os autores, a vulnerabilidade começa a surgir "como ideia subjacente à noção de capacidade de resposta" de um ambiente ou uma população aos processos perigosos (MARANDOLA Jr;; HOGAN, 2004, p. 32).

Já o uso de programas computacionais estatísticos independentes (ou associados a Sistemas de Informação Geográfica) para estudos de vulnerabilidade surge a partir dos finais da década de 1980 e na década de 1990, com destaque para Blaikie et al. (1994) e para Cutter (1996), que se dedicaram ao estudo da vulnerabilidade por meio da análise fatorial de diferentes variáveis e indicadores, considerando as dimensões sociais, econômicas, políticas e culturais que, via de regra, fazem parte dos principais objetos de estudo da Geografia.

Tendo em vista a avaliação da propensão ao risco, ou seja, a vulnerabilidade social, Cutter, Boruff e Shirley (2003) propõem um modelo de construção de um índice que agrega ou sintetiza a informação relevante sobre as características que podem influenciá-la (dados demográficos, como a idade e sexo, a existência de população com necessidades educativas especiais, o letramento, a cultura, a economia). Tratase do SoVI ${ }^{\mathrm{TM}}$, que se obtém após análise fatorial por componentes principais de um conjunto de variáveis censitárias, na escala do município, tidas como representativas das características anteriormente referidas. O modelo foi aplicado e reaplicado nos EUA, usando censos atuais e antigos, fazendo uso de diferentes escalas de análise, como municípios, unidades censitárias, unidades territoriais, sendo também adaptado para países como Noruega e Vietnã, entre outros. A autora ilustra os resultados alcançados para os EUA, tomando por base dados do censo de 2000, no qual o nível mais baixo de vulnerabilidade social aparece na zona costeira oriental, de Washington D.C. até Nova York, incluindo Boston. 0 mesmo acontece nos municípios da costa da Califórnia, áreas consideradas ricas e sem níveis extremos de população envelhecida e/ou feminina. Os níveis mais altos de vulnerabilidade social ganham expressão, por exemplo, ao longo do rio Mississipi, região tradicionalmente pobre e com população predominantemente afro-americana, com pouca oferta de emprego e baixos níveis de escolaridade. A autora conclui o estudo afirmando que:

A ciência da vulnerabilidade fornece a base para a construção de métricas para a vulnerabilidade, que incluem o cálculo de índices para a medição da vulnerabilidade social, assim como modelos geoespaciais para a intersecção dos processos sociais e físicos (CUTTER, 2011, p. 66).

Esta metodologia foi seguida e utilizada também em Portugal, no âmbito do projeto "Vulnerabilidade Social, Estratégias de Planeamento: uma abordagem integrada", desenvolvido no Centro de Estudos Sociais

\footnotetext{
4 Comentário nosso.

5 Comentário nosso.
} 
da Universidade de Coimbra, tendo permitido uma síntese das principais características, com uma leitura em diferentes escalas das condições de vulnerabilidade social a processos perigosos (CUNHA et al., 2010; CUNHA, LEAL, 2012; MENDES et al., 2009, 2011).

Muitos são os estudos que cuidam da integração de dados geoespaciais com dados socioeconômicos, principalmente dados quantitativos, relativos à economia, à sociedade e à demografia, para a obtenção de resultados relativos à avaliação da vulnerabilidade. Nesses casos, deve-se ter em conta a complexidade ou, por outro lado, o caráter redutor que tais dados quantitativos encerram, pois um desafio constante dos pesquisadores para garantir a pertinência dos parâmetros quantitativos passa pela inclusão ou pelo cruzamento destes com dados qualitativos, oriundos de consulta às populações e aos atores das comunidades envolvidas, bem como de observações de campo.

A experiência de Alexander (2011) atrela a vulnerabilidade a uma dialética entre os fatores que aumentam os riscos e aqueles que os diminuem (chamados de fatores de atenuação), bem como a percepção da população e de agentes envolvidos.

Esta dialética é ainda influenciada pela percepção do risco, que tanto pode aumentar como diminuir a vulnerabilidade, dependendo do seu nível de relevância ou de exatidão. Por isso, em termos esquemáticos: Vulnerabilidade total aos desastres = Processos de amplificação do risco - Processos de atenuação do risco \pm Fatores de percepção do risco (ALEXANDER, 2011, p. 14).

Por se tratar de um tema tão complexo, a cultura é difícil de se medir e abarca fenômenos diversos, transforma e é transformada de acordo com as diferentes escalas territoriais e ambientes de convívio na sociedade.

[...] a cultura sofre um processo constante de metamorfose à medida que se adapta às alterações de circunstâncias do mundo moderno e ao modo como somos capazes de o interpretar. Por conseguinte, existem poucas formas confiáveis de medir a cultura. [...] Se se pretende promover a mudança, é mais provável que ela seja bem-sucedida se for compatível com a cultura predominante, ao passo que se for contra ela é provável que o processo adaptativo seja bloqueado por razões aparentemente ilógicas (ALEXANDER, 2011, p. 17).

Pelas bases conceituais aqui apresentadas, observamos que os caminhos para a análise da vulnerabilidade passam por diferentes componentes quantitativos e qualitativos, que se complementam e que não podem ser desprezados. Conhecer o contexto em que se insere o grupo social envolvido, em termos socioeconômicos e ambientais, é o ponto de partida para os estudos de vulnerabilidade, nos quais devem ser também incorporadas a cultura e a percepção da sociedade envolvida, para que seja possível alguma precisão no mapeamento da vulnerabilidade e dos riscos. Só assim será possível nos aproximarmos do indicado pelas Nações Unidas para o enfrentamento, com qualidade, dos riscos de desastres, no sentido de minimizar as perdas e os danos à sociedade, à economia e ao ambiente.

\section{Cartografia das vulnerabilidades socioambientais em países lusófonos: principais influências e estudos para Brasil e Portugal}

Na perspectiva deste artigo serão abordados estudos atuais de vulnerabilidade nos países lusófonos, apoiados, prioritariamente, em trabalhos desenvolvidos no Brasil e em Portugal.

Adotando os princípios metodológicos de Cutter (1996, 2003, 2011) e visando apresentar um índice de vulnerabilidade social aos processos naturais e tecnológicos perigosos para Portugal, baseado na capacidade de resistência e de resiliência de populações e de território, apresentamos os estudos de Mendes et al. (2009) e Cunha et al. (2011), que realizaram uma avaliação prévia da vulnerabilidade social para os concelhos ${ }^{6}$ de Portugal e uma avaliação na escala da freguesia ${ }^{7}$ em sete concelhos da Região Centro

\footnotetext{
6 Concelho: nomenclatura adotada em Portugal para a subdivisão do território sob administração de um presidente da câmara e das restantes entidades autárquicas; divisão administrativa imediatamente inferior à categoria de distrito; município (PORTO EDITORA, 2008).

7 Freguesia: nomenclatura adotada em Portugal para a subdivisão de um concelho, que constitui a menor entidade administrativa (PORTO EDITORA, 2008).
} 
Portuguesa. A Figura 1 apresenta as relações entre risco e vulnerabilidade social, que são a base para o desenvolvimento da metodologia proposta pelos autores.

Como se pode observar pela Figura 1, a perigosidade ou probabilidade de ocorrência de eventos perigosos pode ser mapeada tendo em conta o fator tempo, por meio do acompanhamento dos eventos passados e presentes, bem como da probabilidade da sua repetição no futuro e, também, considerando o fator espaço, ou seja, a suscetibilidade do território para a sua distribuição. 0 mapeamento da perigosidade pode ser feito por meio da produção de mapas temáticos que integrem a susceptibilidade e a probabilidade de ocorrência de eventos perigosos, composta basicamente por mapas físicos, incluindo a delimitação das áreas de diferentes abrangências dos fenômenos em estudo, tendo em conta sua geologia, geomorfologia, declividade, hidrografia, clima, uso da terra, dentre outros.

Já a vulnerabilidade (em sentido amplo) decorre de estudos da vulnerabilidade social ou socioambiental que se apoiam em análises e metodologias estatísticas de dados censitários e no estudo da distribuição da população e dos bens materiais expostos aos eventos perigosos. Para que se efetive o mapeamento e a análise dos riscos, torna-se necessária a combinação dos mapeamentos de perigosidade e de vulnerabilidade.

Para além de uma análise multiescalar, o projeto desenvolvido em Coimbra (MENDES et al., 2009, p. 68), que entende o conceito de vulnerabilidade social associado ao "nível de resiliência ou resistência dos indivíduos e comunidades quando expostos a processos ou eventos danosos", desdobra-se em duas componentes: a criticidade e a capacidade de suporte. A primeira corresponde ao "conjunto de características individuais e comportamentais que podem contribuir para a ruptura do sistema" (MENDES et al., 2009, p. 69), e a segunda diz respeito "ao conjunto de infraestruturas territoriais que permite à comunidade reagir em caso de desastre" (MENDES et al., 2009, p. 70).

A análise dos mapas apresentados para o conjunto do país aponta, claramente, para valores de criticidade e de capacidade de suporte que, genericamente, são pouco correlacionáveis entre si. Ainda assim, sua leitura ressalta claramente a importância das principais regiões metropolitanas do país (Lisboa e Porto), que apresentam valores de criticidade mais baixos e capacidade de suporte mais elevada, logo valores de vulnerabilidade social mais baixos. No mesmo sentido, podem-se ler os valores dos concelhos que integram as principais cidades médias do país (Braga, Coimbra, Santarém, Évora, Beja e Faro, por exemplo). No extremo oposto, ou seja, os concelhos que, por terem níveis de criticidade mais elevada e níveis de capacidade de suporte mais baixa, apontam para índices de mais elevada vulnerabilidade social, são em regra aqueles do interior mais rural, particularmente do norte do país.

Na análise feita em nível do concelho, ou seja, com desagregação no nível das freguesias, ressalta-se a

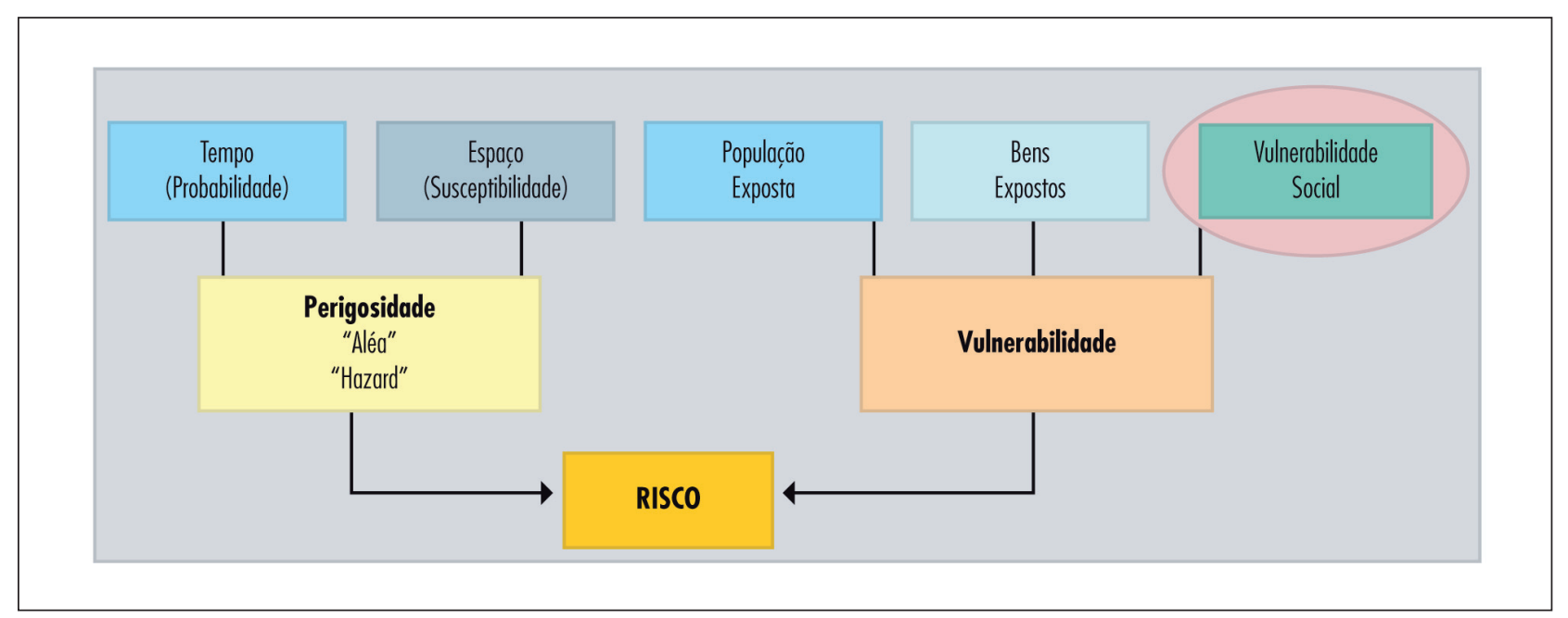

Figura 1 - Vulnerabilidade e risco

Fonte: Adaptado de CUNHA et al., 2011.

urbe. Revista Brasileira de Gestão Urbana (Brazilian Journal of Urban Management), v. 5, n. 1, p. 15-31, jan./jun. 2013 
enorme heterogeneidade interna dos municípios. A análise foi feita para apenas sete concelhos da região e, por exemplo, no caso de Coimbra, o mapa de criticidade ressalta a elevada criticidade das freguesias do Centro Histórico. 0 mapa da Capacidade de Suporte, por sua vez, ressalta a baixa capacidade das freguesias periurbanas e rurais. Isso se traduz, no mapa final, em valores de vulnerabilidade social que se destacam, claramente, em valores mais elevados em freguesias urbanas do Centro Histórico e da periferia e valores mais baixos nas freguesias urbanas mais ricas, com melhores infraestruturas e de maior procura por parte da população.

Para além das questões de escala, outra questão se coloca quando se passa da escala nacional para a escala municipal e tem a ver com o tipo de unidades administrativas ou cartográficas que podem ser utilizadas. Nos estudos em escala nacional, a utilização dos concelhos como unidades administrativas de referência parece não oferecer dúvidas, já que estes correspondem a unidades territoriais que, embora naturalmente diversas, apresentam estruturas semelhantes e permitem comparações com lógicas territoriais, econômicas e socioculturais, logo de vulnerabilidade (CUNHA et al., 2010); no entanto, a descida em nível local, ou seja, no interior dos concelhos, levanta outros tipos de problemas.

Por um lado, existe uma enorme diversidade no número e na tipologia das freguesias em cada concelho. Em alguns deles o número de freguesias é tão baixo que não permite comparações significativas em nível de concelho. Noutros, o número é demasiado elevado e os contrastes se tornam complexos. Por outro lado, com as freguesias não se atinge o nível de discriminação territorial conseguido com os mapas de suscetibilidade/perigosidade, baseados em dados dos fatores de perigosidade trabalhados em escala do pixel definido para o projeto cartográfico. Isso porque, quando se pretende maior nível de pormenor tratando a informação em nível da seção ou da subseção estatística, deparamo-nos com o fato de essas unidades não apresentarem qualquer tipo de coerência territorial com lógica de planejamento. Assim, torna-se necessário criar novas unidades territoriais, com coerência e homogeneidade internas, mas que permitam utilizar os dados estatísticos em nível das unidades de maior detalhe e pormenor, ou seja, da subseção estatística.
Uma recente experiência (CUNHA et al., 2010) realizada num pequeno município português (Torres Novas) permitiu aplicar essa metodologia com sucesso e diferenciar os tipos de riscos naturais do espaço urbano do Município, em regra com valores de vulnerabilidade social mais baixos do que nos espaços rurais, particularmente os pequenos aglomerados, nos quais o envelhecimento da população, a menor capacidade econômica e as más condições das edificações são responsáveis, entre outros fatores, por elevados valores de vulnerabilidade social.

Dentre os autores que têm se dedicado a estudos de vulnerabilidade no território brasileiro, encontram-se Garcia e Matos (2007), que avaliaram as condições de vida das famílias brasileiras e realizaram o mapeamento da sua distribuição espacial, tendo como base os microdados do Censo Demográfico de 2000. O estudo permitiu a construção de um indicador de vulnerabilidade social das famílias, denominado Índice de Vulnerabilidade Municipal (IVM), que leva em consideração informações sobre o grau de inserção educacional, o grau de inserção econômica local e o grau de inserção habitacional. Os resultados alcançados e as comparações efetuadas com o Índice de Desenvolvimento Humano (IDH) mostraram-se convincentes e validaram o indicador adotado, o qual se revelou robusto e capaz de apontar diferenças intermunicipais e intramunicipais no tocante à vulnerabilidade social.

0 estudo levou os autores a concluir que o índice adotado é sensível às condições de vida e de desenvolvimento das localidades e regiões, o que lhe confere a característica de indicador socioespacial. As representações cartográficas temáticas foram utilizadas para ilustrar a vulnerabilidade social em escala nacional (municípios do Brasil) e municipal (bairros que compõem a Região Metropolitana de Belo Horizonte - MG). Em nível nacional, os resultados alcançados pelos autores apontam alta vulnerabilidade em cerca de 35\% dos municípios brasileiros, com destaque para municípios da Região Nordeste, bem como da Região Norte, embora apareçam também municípios com alta incidência de população vulnerável em áreas rurais do nordeste do estado de Minas Gerais, além de outras no interior dos estados do Paraná e de Santa Catarina. Os autores destacam, ainda, a existência de áreas rurais pouco vulneráveis no eixo que liga São Paulo a Brasília, bem 
como na região norte do estado do Rio Grande do Sul (GARCIA; MATOS, 2007).

Outro exemplo de pesquisa brasileira, agora na escala local/regional, fez uso de geoprocessamento em estudo de vulnerabilidade para a metrópole paulistana e foi realizado por Alves (2006), sendo que nele a categoria vulnerabilidade socioambiental foi estudada por meio de indicadores sociais e ambientais, na escala mais desagregada possível, ou seja, a dos setores censitários. Em sua metodologia de trabalho propôs uma tipologia simples, em que os setores censitários do município de São Paulo são classificados em quatro categorias de vulnerabilidade, resultantes da combinação das duas dimensões - risco ambiental (proximidade dos cursos d'água) e degradação ambiental (baixa cobertura de esgoto). 0 autor realizou a análise utilizando a escala do município e outra mais detalhada, considerando um setor da Zona Leste da cidade de São Paulo. Dentre os resultados apresentados destaca-se o fato de, dentro do grupo de alta vulnerabilidade, aparecerem diferenças significativas nas condições socioeconômicas e demográficas relacionadas às diversas categorias de vulnerabilidade socioambiental. Em alguns casos, a vulnerabilidade social é agravada por situações de risco e de degradação ambiental, com condições socioeconômicas significativamente piores, além de maior concentração de crianças e jovens, do que aquelas com menor grau de vulnerabilidade ambiental. 0 autor detectou, ainda, áreas críticas, nas quais há forte concentração de problemas e riscos sociais e ambientais, com maiores níveis de população pobre e com privação social, tendo, portanto, menor capacidade de reação às manifestações de risco.

Também nessa escala de análise, o trabalho de Fonseca Alves et al. (2010) trata da aplicação de um modelo de vulnerabilidade socioambiental, no contexto das mudanças climáticas, para municípios do litoral paulista. 0 trabalho apresenta resultados relativos à vulnerabilidade socioambiental, considerando variáveis socioeconômicas associadas com os setores censitários de renda, escolaridade, atendimento pela rede de esgotos, rede de água e coleta de resíduos sólidos, o chamado Índice Paulista de Vulnerabilidade Social (IPVS), e variáveis ambientais relativas à altimetria e à declividade. Nele foi usado o modelo de estimativa Kernel, técnica de análise espacial baseada na criação de superfícies de densidade, para mostrar a distribuição espacial das áreas de vulnerabilidade socioambiental. Dentre os resultados apresentados por meio de representações cartográficas, estão aqueles indicando as condições de saneamento básico dos municípios do litoral paulista: adequadas com respeito ao acesso à rede de água e à coleta de resíduos sólidos (predomínio de mais de $80 \%$ de atendimento de água e coleta de resíduos sólidos), mas com problemas relacionados ao esgoto (predomínio de valores abaixo de $50 \%$ de atendimento pela rede geral de esgotos).

Quanto à escolaridade, os autores observaram que ocorre maior proporção de pessoas com 4 a 10 anos de escolaridade (49,6\%), em relação ao total de pessoas responsáveis pelos domicílios, no conjunto do litoral paulista. Quanto à renda, com exceção do município de Santos, a maior parte dos municípios concentra pelo menos mais do que $30 \%$ de pessoas com renda igual ou inferior a dois salários mínimos. 0 mapa de distribuição da vulnerabilidade aponta áreas de risco de deslizamento, as quais estão associadas a um perfil socioeconômico de baixa renda e com baixo nível de escolaridade. No entanto, algumas dessas áreas são caracterizadas por um perfil de setores com média ou alta renda e elevado grau de escolaridade como, por exemplo, as localizadas no município de Ilhabela. Nesse sentido, advertem os autores:

Assim, esses resultados sugerem que uma parcela significativa da população residente nos municípios do litoral paulista, seja ela de baixa, média ou alta renda, está em situação de alta ou muito alta vulnerabilidade socioambiental, ainda que com graus diferenciados de susceptibilidade e exposição ao risco ambiental (FONSECA ALVES et al., 2010, p. 18).

Tal consideração aponta para uma questão fundamental, quando se abordam aspectos associados à vulnerabilidade e riscos socioambientais na faixa litorânea: diante da complexidade da urbanização das cidades brasileiras, em especial na faixa litorânea, mas não exclusivamente, cujas construções habitacionais e de veraneio chegam muitas vezes antes da infraestrutura básica, é comum se encontrarem residências instaladas em áreas muitas vezes impróprias. Nesses casos, predominam instalações habitacionais em vertentes 
com alta declividade, muitas vezes sem cobertura do solo, em áreas com forte probabilidade de inundação devido à densa drenagem e à presença de chuvas torrenciais em certas épocas do ano, colocando em risco seus moradores, independentemente da classe social.

0 estudo de Almeida (2011) propõe uma Geografia dos riscos e das vulnerabilidades, tendo como base uma ampla discussão teórica sobre tais conceitos, com ênfase no ambiente das cidades. Além de discutir os conceitos de risco, perigosidade e vulnerabilidade na história recente - a partir da década de 1980 -, o autor apresenta os resultados de mensuração de vulnerabilidades socioambientais de rios urbanos no Brasil, com aplicação na bacia hidrográfica do rio Maranguapinho, localizado na Região Metropolitana de Fortaleza. Nesse caso, o mapa de vulnerabilidade socioambiental resulta da integração de mapas derivados do Índice de Vulnerabilidade Social (IVS) e do Índice de Vulnerabilidade Físico-Espacial às Inundações (IVFI). Tais índices permitiram "a identificação e localização dos espaços onde ocorre coincidência de riscos e vulnerabilidades - sociais e ambientais - resultando num Índice de Vulnerabilidade Socioambiental - IVSA para bacia hidrográfica do rio Maranguapinho" (ALMEIDA, 2011, p. 95). Por meio desse estudo, o autor pôde observar a formação de grupos homogêneos de vulnerabilidade socioambiental, identificando espaços em que ocorrem vulnerabilidades sociais e ambientais.

Os resultados dos estudos aqui apresentados apontam para a prevalência de alguma convergência teórica entre os estudos de vulnerabilidade socioeconômica e ambiental dos dois países lusófonos. A escala de trabalho, via de regra, parte do geral e detalha amostras no particular, tendo as publicações transitado entre as escalas nacional, regional e local, em seus estudos de caso. Independentemente das designações formais utilizadas, todos os autores estudados concentram-se na definição de índices de vulnerabilidade levando em conta as condições sociais, econômicas e ambientais como, por exemplo, habitação, educação, renda, infraestrutura urbana e saneamento. De maneira geral, os resultados apresentados salientam a importância desse tipo de análise para a adequação das políticas públicas e para a organização territorial, com vistas a apontar a maior ou menor resistência e resiliência das comunidades aos desastres naturais.

\section{Vulnerabilidade socioambiental de municípios selecionados no estado de São Paulo, Brasil e na Região Centro de Portugal}

Para a realização de um estudo de caso em escala regional, considerando as bases metodológicas apresentadas anteriormente e sua aplicação às realidades do Brasil e de Portugal, selecionamos 17 concelhos na Região Centro de Portugal e 20 municípios localizados no estado de São Paulo, Brasil. A localização das áreas de estudo é apresentada nas Figuras 2 e 3, respectivamente.

Os 17 concelhos de Portugal ocupam uma superfície territorial de $4.841 \mathrm{~km}^{2}$ e abrigam um total de 731.847 habitantes, com populações variando de 13.295 habitantes (Mira) a 131.446 habitantes (Coimbra), de acordo com o censo publicado pelo INE para 2011.

No Brasil, os 20 municípios paulistas em estudo compõem as regiões metropolitanas de São Paulo e de Santos, e regiões administrativas de Sorocaba e Registro. Ocupam uma superfície territorial de 6.774 $\mathrm{km}^{2}$ e abrigam um total de 1.990 .071 habitantes, com populações variando de 10.303 habitantes (Pedro de Toledo) a 596.060 habitantes (Sorocaba), de acordo com o censo publicado pelo IBGE (2012).

Esta pesquisa correspondeu à parte principal da atividade de pós-doutorado desenvolvida na Universidade de Coimbra, sob o tema Geotecnologias aplicadas na Análise da Vulnerabilidade Social e Ambiental: um estudo metodológico comparativo entre Portugal e Brasil (FREITAS, 2012), cujos resultados preliminares foram divulgados em eventos científicos em Portugal e no Brasil, como pode ser verificado nos trabalhos de Ramos et al. (2012); Freitas e Cunha (2012a, b).

Por meio da análise fatorial relativa à criticidade e capacidade de suporte, avalia-se a vulnerabilidade socioambiental das áreas estudadas, com vistas a observar o desempenho do modelo no contexto da realidade de cada uma das regiões.

Para a análise da criticidade, realizamos um levantamento dos dados censitários concernentes aos aspectos da vida da população, na busca de características dos concelhos portugueses e dos 


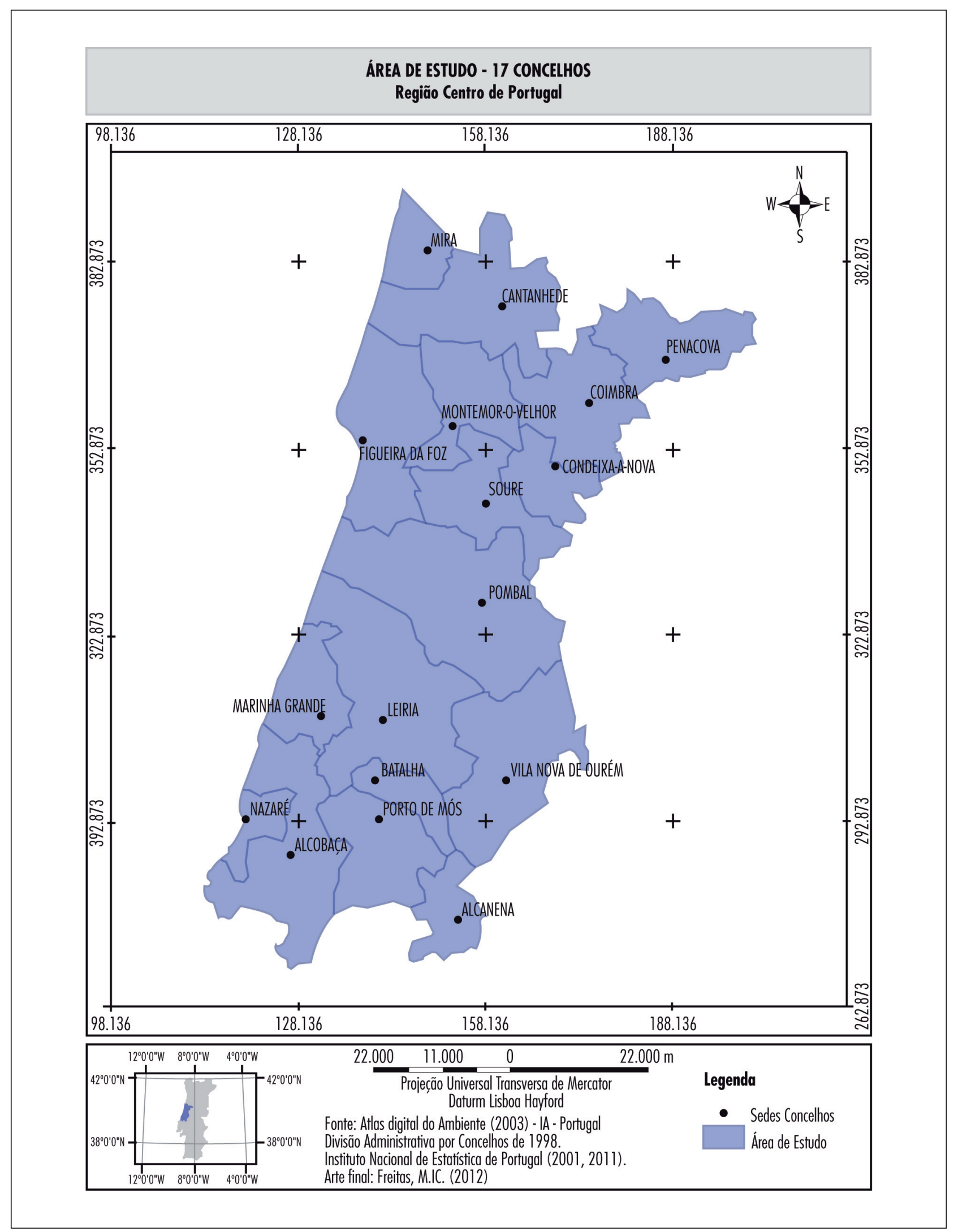

Figura 2 - Área de estudo referente aos 17 Concelhos da Região Centro de Portugal Fonte: FREITAS, 2012. 


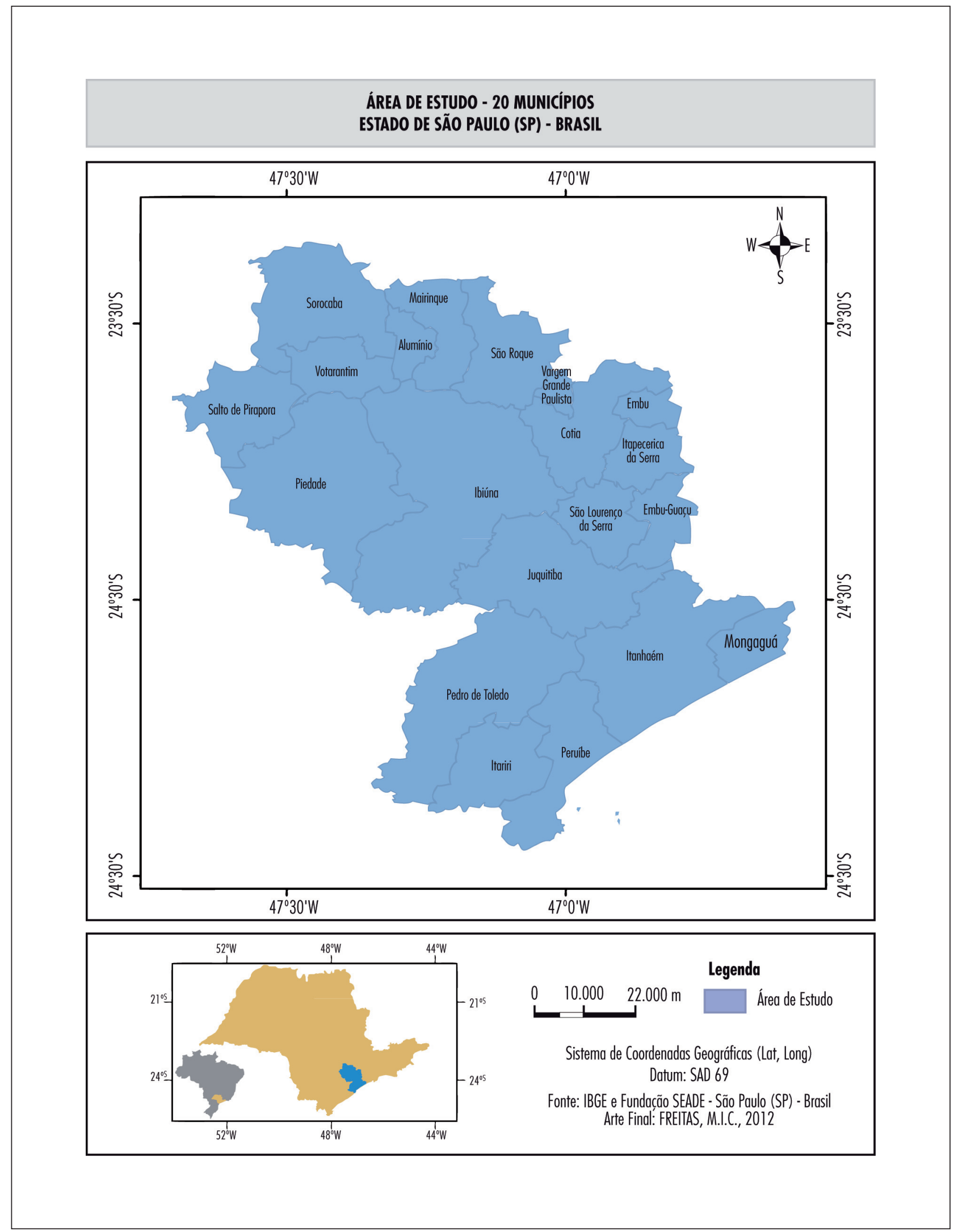

Figura 3 - Área de estudo no estado de São Paulo, Brasil Fonte: FREITAS, 2012. 
municípios brasileiros que dessem indicativos de ruptura do sistema, no caso de eventos perigosos associados a riscos naturais ou tecnológicos.

No caso de Portugal foram selecionadas, inicialmente, 108 variáveis. Da execução do modelo de análise fatorial resultaram 21 variáveis explicativas, que foram agregadas em cinco fatores, os quais detêm $76 \%$ da variância acumulada, considerando-se os 17 concelhos em estudo. As comunalidades das variáveis foram todas superiores a 0,88.

De posse de tais dados, exportamos para o ArcGIS os resultados relativos aos cinco fatores mais significativos para cada concelho em estudo, realizando sua espacialização tendo como referência os limites dos concelhos do Atlas do Ambiente em formato digital. A sua representação temática foi feita por meio da classificação de seus resultados, aplicando, numa primeira aproximação, o classificador Natural Breaks (Jenks), seguido de classificação manual.

No caso brasileiro, foi adotado um procedimento similar, no qual foram selecionadas, inicialmente, 75 variáveis que, após redução pela análise fatorial, resultaram em 18 variáveis explicativas, agregadas em cinco fatores que detêm $67 \%$ da variância acumulada, considerando os 20 municípios em estudo. As comunalidades das variáveis foram superiores a 0,78.

Em procedimento análogo, realizamos o estudo da capacidade de suporte dos 17 concelhos em estudo, levantando os dados censitários que indicassem o nível de infraestruturas territoriais que permitem a reação das comunidades em caso de desastre associado a risco natural ou tecnológico.

Da execução do modelo de análise fatorial resultaram 16 variáveis explicativas, dentre as quais foi possível selecionar cinco fatores, que detêm $68 \%$ da variância acumulada, considerando os 17 concelhos em estudo no caso português. As comunalidades das variáveis foram sempre superiores a 0,83, o que aponta para o seu contributo significativo para a formação dos fatores.

No caso do Brasil, foram selecionadas 54 variáveis, com redução por análise fatorial para 17 variáveis explicativas, agregadas em cinco fatores, que detêm 68\% da variância acumulada, considerando os 20 municípios em estudo. As comunalidades das variáveis foram sempre superiores a 0,706, o que aponta, também nesse caso, para um significativo contributo na formação dos mesmos.
Feitos os cálculos das componentes criticidade e capacidade de suporte, para Portugal e Brasil, foi possível o cálculo da Vulnerabilidade Socioambiental, tendo em conta a expressão matemática (1):

$$
\mathrm{VS}=\mathrm{C} \times \mathrm{CS}
$$

Sendo: VS = Vulnerabilidade Socioambiental; $\mathrm{C}=$ Criticidade; CS = Capacidade de Suporte.

Com base nesses elementos, foi possível a elaboração de mapas temáticos e gráficos ilustrativos da vulnerabilidade socioambiental e de seus componentes.

\section{Resultados do estudo das vulnerabilidades no Brasil e em Portugal: convergências e divergências}

Com base no procedimento descrito, foi possível a elaboração de tabelas e mapas temáticos ilustrativos da vulnerabilidade socioambiental e dos seus componentes para as áreas de estudo em Portugal e no Brasil.

No caso de Portugal, os principais fatores relativos à criticidade correspondem aos fatores 1 e 2 . 0 fator 1 , relacionado com a economia e as condições de vida da população, explica $26 \%$ da variância e dá indicativo dos concelhos com maior acesso ao crédito para habitação e ao emprego; o fator 2 , que diz respeito à população e ao grau de proteção social que aufere, responde por $18 \%$ da variância total e dá destaque para as baixas condições de vida e baixa natalidade. 0 fator 3 (13\% da variância) apresenta o baixo poder de compra da população e o acesso às telecomunicações; já o fator 4 (10\% da variância) indica as transformações no meio rural enfatizando as áreas sem uso agrícola. Por fim, o fator 5 ( $9 \%$ da variância) apresenta variável relacionada à evasão escolar.

Quanto à capacidade de suporte, observamos que as principais variáveis explicativas do fator 1, que concentram $20 \%$ da variância, estão relacionadas com as condições econômicas; essas variáveis apontam os conselhos com maior desenvolvimento de acordo com o número de empresas e estabelecimentos bancários, bem como a proporção de famílias em alojamentos unifamiliares e, em contraponto, os deficit das corporações de bombeiros para o atendimento das demandas regionais. Os fatores 2, 3, 4 e 5 estão associados, predominantemente, às condições dos alojamentos e 
dos edifícios; o fator 2 (14\% da variância) destaca a falta de conservação dos edifícios, o fator 3 $(13 \%)$ indica a presença de edifícios vagos e com necessidade de reparação na área urbana, o fator $4(14 \%)$ associa-se à baixa quantidade de edifícios novos e o fator $5(8 \%)$ indica a sazonalidade no uso de parcela dos alojamentos.

A Figura 4 mostra a representação espacial dos resultados obtidos no que se refere à vulnerabilidade socioambiental dos 17 concelhos de Portugal.

Os resultados indicam como principais fatores de vulnerabilidade socioambiental as condições relativas ao baixo dinamismo econômico e aos deficit demográficos, principalmente relacionados com a população infanto-juvenil e com o êxodo rural. Esse é um indicador do envelhecimento da população, o que é recorrente nos concelhos portugueses, e que se confirma para a área de estudo, sobretudo nos concelhos de menor peso urbano. Os valores altos ou muito altos de vulnerabilidade aparecem nos concelhos do setor norte (Montemor-o-Velho, Soure, Mira e Penacova), com menor desenvolvimento econômico e com condições ambientais e infraestruturas menos desenvolvidas para enfrentar situações de risco. Também com esse perfil estão alguns concelhos do setor sul da área de estudo, como Alcanena e Porto de Mós.

No caso do Brasil, a análise dos fatores relativos à criticidade indica que o fator 1 , relacionado com a demografia e com o investimento no campo, explica $22 \%$ da variância. Esse fator indica o predomínio da população jovem nos municípios, por meio de variáveis negativas para a população com mais de 65 anos, associadas com a taxa de natalidade que aparece com valores positivos. 0 fator 2 , que responde por $16 \%$ da variância total, diz respeito à violência urbana e às condições de saúde, dando destaque para as ocorrências de furtos consumados, tráfico de drogas, crimes e mortes pela Síndrome de Imunodeficiência Adquirida (SIDA), ou AIDS, como é mais conhecida no Brasil. Os fatores 3, 4 e 5 apresentam variáveis associadas à condição de vida, educação e demografia, com o fator 3 (14\% da variância) sendo expresso pela presença de crianças e adolescentes, incidência de pobreza e evasão escolar, o fator $4(8 \%)$ destaca a reprovação escolar e a taxa de natalidade, complementados pelo fator $5(7 \%)$, que indica a existência de moradias em áreas de risco.

Quanto à capacidade de suporte, observamos que as principais variáveis explicativas do fator 1 , o qual concentra $21 \%$ da variância, estão relacionadas com as transformações econômicas e habitacionais do meio rural para o urbano, dando indicação da diminuição dos investimentos econômicos relacionados com as atividades agropecuárias, associados ao aumento da taxa de urbanização das cidades. 0 fator 2 , que detém $15 \%$ da variância, apresenta o aumento de alojamentos do tipo apartamento e a infraestrutura em saúde relacionada à presença de médicos e dentistas com registro. Os fatores 3, 4 e 5 destacam variáveis associadas ao desenvolvimento regional, à infraestrutura e às condições dos alojamentos; o fator 3 (14\% da variância) indica o Produto Interno Bruto associado às indústrias e serviços, e a presença de domicílios com espaço suficiente, inclusive aqueles denominados domicílios coletivos; o fator 4 (10\%) corresponde à presença de leitos hospitalares e à existência de favelas; e o fator 5 (8\%) indica domicílios vagos nas áreas urbanas.

Na Figura 5, apresenta-se a representação espacial dos resultados obtidos no que se refere à vulnerabilidade socioambiental dos 20 municípios do estado de São Paulo.

Os resultados indicam como principais fatores de vulnerabilidade socioambiental as condições relativas ao menor desenvolvimento econômico e às limitadas condições de infraestruturas e desempenho ambiental em face de situações de risco. Os municípios com vulnerabilidade elevada ou muito elevada aparecem no interior, na região serrana, de economia predominantemente rural, ainda que mitigada pela sua associação ao turismo rural, como Ibiúna, Embu-Guaçu, Juquitiba e Piedade.

0 estudo indicou como principais fatores de vulnerabilidade socioambiental, no contexto português, a contração econômica de parte dos concelhos, a baixa natalidade, o envelhecimento da população e da infraestrutura habitacional; parte desses fatores se associa ao abandono do meio rural. No caso brasileiro, os principais fatores de alta vulnerabilidade estão relacionados à economia e às infraestruturas para o enfrentamento de eventos perigosos. Ambos os resultados dão indicações da preponderância dos fatores econômicos e sociais na diferenciação territorial da vulnerabilidade, distinguindo regiões com diferentes graus de vulnerabilidade socioambiental, ou seja, com populações e territórios mais ou menos providos de condições para o enfrentamento e 


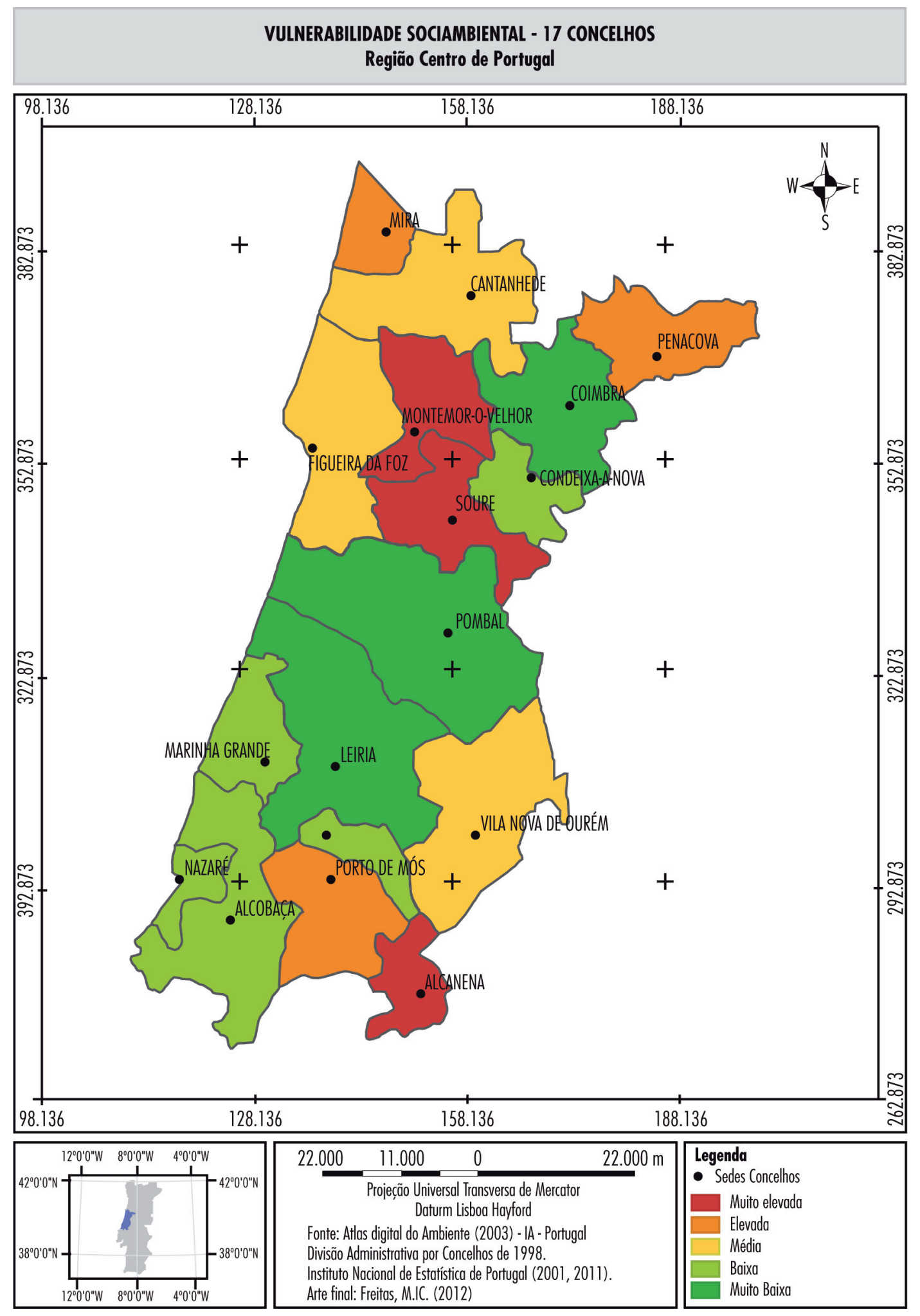

Figura 4 - Vulnerabilidade Socioambiental dos 17 concelhos da Região Centro de Portugal Fonte: FREITAS, 2012. 

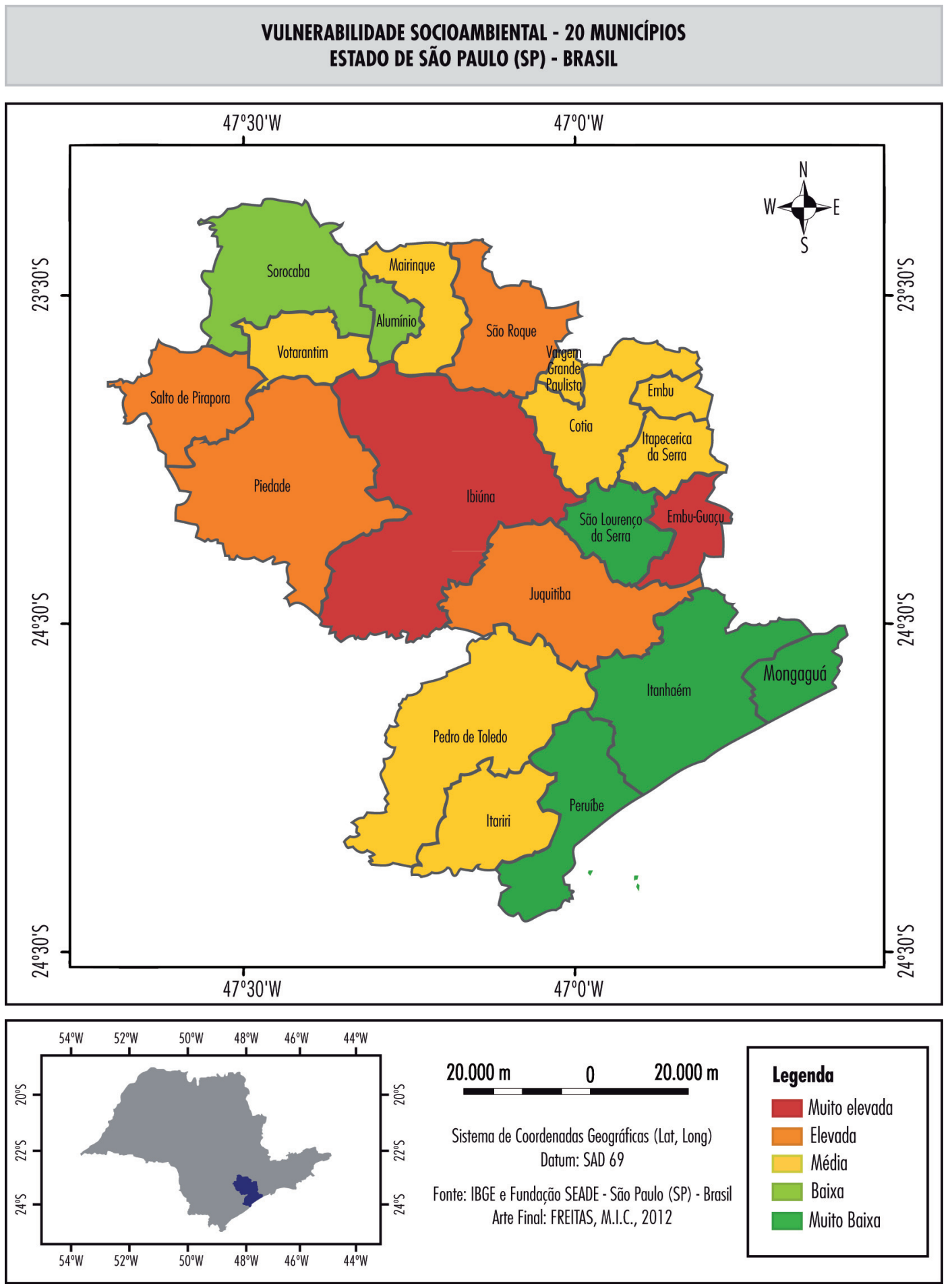

Figura 5 - Vulnerabilidade socioambiental dos 20 municípios do estado de São Paulo Fonte: FREITAS, 2012. 
recuperação de manifestações de riscos, sejam eles naturais, tecnológicos ou mistos.

No caso brasileiro, os contrastes sociais, embora minimizados, em vista da situação econômica favorável da atualidade, ainda se fazem presentes nos itens relativos à violência, deficiências na educação e taxas de pobreza.

\section{Considerações finais}

Dentre as convergências metodológicas em termos mundiais, das quais não se distanciam os países lusófonos em estudo, observamos que a base dos estudos de vulnerabilidade se apoia em análises quantitativas, com forte ênfase para modelos estatísticos, associados a Sistemas de Informação Geográfica. Tendo em consideração as conhecidas limitações dos métodos quantitativos, verifica-se hoje uma tendência em inserir, ou pelo menos validar, esses modelos com variáveis qualitativas como as relacionadas com a percepção das populações e dos gestores no que se refere à vulnerabilidade e aos riscos de desastres; por esta perspectiva, parte-se do pressuposto de que a realidade socioeconômica, ambiental e as raízes culturais ditam comportamentos diferenciados no enfrentamento de situações de riscos de desastres.

Outra convergência observada diz respeito ao uso de diferentes escalas de análise, o que se observa pela busca, dos autores consultados, de um contraponto entre o global e o local, refletido nos estudos feitos em escalas dos países, das regiões ou até dos municípios, setores censitários, bairros ou outras divisões intraurbanas.

As divergências residem nos tipos de variáveis selecionadas e nas metodologias de tratamento, em termos dos aspectos sociais, ambientais e físicos que as influenciam. Muitos dos trabalhos buscam realizar recortes na grande gama de variáveis disponíveis, selecionando-as previamente ou usando algoritmos que permitam essa seleção através de critérios estatísticos. Seja como for, notamos a não padronização e a dificuldade de colocar, sob um único modelo, realidades territoriais tão díspares em termos de ambiente, economia, sociedade e, mesmo, cultura.

No intuito de aplicar um dos modelos de vulnerabilidade, considerando as diferentes realidades de Portugal e do Brasil, em termos de dimensão territorial, economia, sociedade e cultura, foram selecionadas duas áreas que se destacam nos respectivos contextos nacionais e estaduais pelo desenvolvimento econômico e pela concentração populacional, nas quais observamos também convergências e divergências.

Na aplicação do modelo, foi possível observar que fatores preponderantes, como a retração econômica de Portugal, o envelhecimento da população e a baixa natalidade estiveram presentes como prioritários na indicação da vulnerabilidade. No caso brasileiro, os fatores dominantes também se relacionam com a economia e a população, principalmente os que expressam as desigualdades socioeconômicas, alta natalidade, violência e baixa escolaridade. Em ambas as regiões ocorrem o abandono de áreas rurais, a desaceleração da vida no campo e a concentração urbana, dando mostras de que o modelo de sociedade no qual estamos inseridos faz com que tendências sejam mantidas, independentemente dos países e dos continentes nos quais desenvolvemos nossas vidas. No estudo de caso, detectamos uma possibilidade de padronização da análise entre os dois países, que poderá ser assumida em futuras análises de vulnerabilidade e riscos, cujos comportamentos tendem a ser mais facilmente mensurados, independentemente do local em que estejamos inseridos.

\section{Referências}

ALEXANDER, D. Modelos de vulnerabilidade social a desastres. Revista Crítica de Ciências Sociais, v. 93, n. 1, p. 9-29, jun. 2011.

ALMEIDA, L. Q. Por uma ciência dos riscos e vulnerabilidades na geografia. Mercator, v. 10, n. 23, p. 83-99, set./ dez. 2011. doi:10.4215/RM2011.1023.0007.

ALVES, H. P. F. A. Vulnerabilidade socioambiental na metrópole paulistana: uma análise sociodemográfica das situações de sobreposição espacial de problemas e riscos sociais e ambientais. Revista Brasileira de Estudos da População, v. 23, n. 1, p. 43-59, jan./jun. 2006. doi:10.1590/S0102-30982006000100004.

BLAIKIE, P. et al. At risk - natural hazards, people's vulnerability and disasters. Londres: Routledge, 1994.

CUNHA, L. et al. Vulnerabilidade, riscos naturais e ordenamento do território. Exemplos no município de Torres Novas. In: COLÓQUIO IBÉRICO DE GEOGRAFIA, 12., 2010, Porto. Anais... Porto: Universidade do Porto, 2010. 
CUNHA, L. et al. Construção de modelos de avaliação de vulnerabilidade social a riscos naturais e tecnológicos. O desafio das escalas. In: SANTOS, N.; CUNHA, L. (Org.). Trunfos de uma geografia activa. Coimbra: Imprensa da Universidade de Coimbra, 2011. p. 627-637.

CUNHA, L.; LEAL, C. Natureza e sociedade no estudo dos riscos naturais. Exemplos de aplicação ao ordenamento do território no município de Torres Novas (Portugal). In: PASSOS, M. M.; CUNHA, L.; JACINTO, R (Org.). As novas geografias dos países de língua portuguesa: paisagens, territórios e políticas no Brasil e em Portugal (II). São Paulo: Geografia em Movimento, 2012. p. 47-66.

CUTTER, S. L. Vulnerability to environmental hazards. Progress in Human Geography, v. 20, n. 4, p. 529-539, 1996. doi:10.1177/030913259602000407.

CUTTER, S. L.; BORUFF, J.; SHIRLEY, W. Social vulnerability to environmental hazards. Social Science Quarterly, v. 84, n. 2, p. 242-261, 2003. doi:10.1111/1540-6237.8402002.

CUTTER, S. L. A ciência da vulnerabilidade: modelos, métodos e indicadores. Revista Crítica de Ciências Sociais, v. 93, n. 1, p. 59-70, jun. 2011.

FONSECA ALVES, H. P. et al. Vulnerabilidade socioambiental nos concelhos do litoral paulista no contexto das mudanças climáticas. In: ENCONTRO NACIONAL DE ESTUDOS POPULACIONAIS, 17., 2010, Caxambu. Anais... Caxambu: ABEP, 2010. p. 1-3. v. 1.

FREITAS, M. I. C. Geotecnologias aplicadas na análise da vulnerabilidade social e ambiental: um estudo metodológico comparativo entre Portugal e Brasil. Rio Claro, 2012. Relatório Final de Estágio de Pós-Doutorado no Exterior - CNPq, Universidade Estadual Paulista.

FREITAS, M. I. C.; CUNHA, L. Vulnerabilidade socioambiental de Concelhos da Região Centro de Portugal por meio de sistema de informação geográfica. In: COLÓQUIO DE GEOGRAFIA DE COIMBRA, 7., 2012, Coimbra. Anais... Coimbra: Universidade de Coimbra. 2012a. 1 CD-ROM.

FREITAS, M. I. C.; CUNHA, L. Vulnerabilidade socioambiental como subsídio para a prevenção de riscos: modelação aplicada à escala municipal em Portugal e no Brasil. In: ENCONTRO NACIONAL DE RISCO, 7.; FÓRUM SOBRE RISCOS E SEGURANÇA DO ISCIA, 1., 2012, Aveiro. Anais... Aveiro: FEDRAVE: Mare Liberum, 2012b. p. 47.
GARCIA, R. A.; MATOS, R. A distribuição espacial da vulnerabilidade social das famílias brasileiras. In: SEMINÁRIO POPULAÇÃO, POBREZA E DESIGUALDADE, 2007, Belo Horizonte. Anais... Belo Horizonte: UFMG, 2007. p. 1-20.

INSTITUTO BRASILEIRO DE GEOGRAFIA E ESTATÍSTICAS IBGE. Censo 2010. 2012 [?]. Disponível em: <http://www. censo2010.ibge.gov.br/>. Acesso em: 23 set. 2012.

MARANDOLA Jr., E.; HOGAN, D. Vulnerabilidades e riscos: entre geografia e demografia. In: ENCONTRO NACIONAL DE ESTUDOS POPULACIONAIS, 14., 2004, Caxambu. Anais... Campinas: ABEP, 2004. Disponível em: <http:// www.abep.org.br>. Acesso em: 23 set. 2012.

MENDES, J. M. et al. Vulnerabilidade social aos riscos naturais e tecnológicos em Portugal. In: SOARES, C. G. et al. (Org.). Riscos industriais e emergentes. Lisboa: Edições Salamandra, 2009. p. 67-84.

MENDES, J. M. et al. A vulnerabilidade social aos perigos naturais e tecnológicos em Portugal. Revista Crítica de Ciências Sociais, v. 93, p. 95-18, jun. 2011.

PORTO EDITORA. Dicionário da língua portuguesa. Porto: Porto Editora, 2008. Disponível em: <http://www. portoeditora.pt/espacolinguaportuguesa/dol/dicionarios-online/>. Acesso em: 20 out. 2012.

RAMOS, A. et al. Cartografia de suscetibilidade a deslizamento e unidades territoriais de risco à escala regional (Figueira da Foz - Nazaré). In: COLÓQUIO DE GEOGRAFIA DE COIMBRA, 7., 2012, Coimbra. Anais.... Coimbra: Universidade de Coimbra, 2012.

REBELO, F. Geografia física e riscos naturais. Coimbra: Imprensa da Universidade de Coimbra, 2010.

UNITED NATIONS - INTERNATIONAL STRATEGY FOR DISASTER REDUCTION - UN-ISDR. Hyogo Framework for Action 2005-2015. 2005. Disponível em: <http://www.unisdr.org/we/coordinate/hfa $>$. Acesso em: 24 jun. 2012.

UNITED NATIONS - INTERNATIONAL STRATEGY FOR DISASTER REDUCTION - UN-ISDR. Global Plataform 2011. 2009. Disponível em: <http://www.unisdr.org/>. Acesso em: 10 ago. 2010.

Recebido: 30/05/2012

Received: 05/30/2012

Aprovado: 18/10/2012

Approved: 10/18/2012 